\title{
LIBYA ANTIQUA
}

The annual review of the Department of Antiquities is the major journal for the publication of reports and articles concerning the archaeology of Libya. Earlier issues can be purchased from:-

L'Erma di Bretschneider, Via Cassiadoro, 19, Rome, Italy.

Prices of the volumes vary but are always reasonable, and all details can be obtained from the distributor in Rome. 


\section{The Society for Libyan Studies}

President:

Vice-President:

Chairman:

Hon. Treasurer:

Secretary:
Officers and Council 1977-78

*Sir Roderick Sarell, K.C.M.G., K.C.V.O.

*Sir Duncan Cumming, K.B.E., C.B.

*J. A. Allan Esq., Ph.D., F.R.G.S. (Royal Geographical Society)

*T. L. Taylor Esq.

*Mrs. S. K. Strong.

\section{Council}

M. Brett Esq., Ph.D.

J. Boardman Esq., F.B.A. (British School at Athens)

*J. P. Carswell Esq., C.B. (British Academy)

Sir Duncan Cumming, K.B.E., C.B.

Professor J. D. Evans, Ph.D., F.S.A. (London University, Institute of Archaeology)

G. Fehérvári Esq., Ph.D.

Professor W. B. Fisher, Dr. de l'Univ.

Mrs. O. P. F. Hackett, O.B.E., F.S.A.

P. M. Kenrick Esq.

*J. A. Lloyd Esq., Ph.D.

*Professor C. B. M. McBurney, Ph.D., Sc.D., F.B.A. (Prehistoric Society)

Miss J. M. Reynolds, F.S.A. (British School at Rome)

A. H. Slater Esq., (Egypt Exploration Society)

J. B. Ward-Perkins Esq., C.M.G., C.B.E., F.B.A., F.S.A.

Professor J. J. Wilkes, Ph.D., F.S.A.

*Members of the Executive Committee 